Citation: Yaslan, H . "Numerical Solution of Fractional Riccati Differential Equation via Shifted Chebyshev Polynomials of the Third Kind". Journal of Engineering Technology and Applied Sciences 2 (1) 2017 : 1-11

\title{
NUMERICAL SOLUTION OF FRACTIONAL RICCATI DIFFERENTIAL EQUATION VIA SHIFTED CHEBYSHEV POLYNOMIALS OF THE THIRD KIND
}

\author{
Handan Cerdik Yaslan \\ Department of Mathematic, Faculty of Arts and Sciences, Pamukkale University, Turkey \\ hcerdik@epau.edu.tr
}

\begin{abstract}
In this paper, Chebyshev collocation method is applied to fractional Riccati differential equation (FRDE) using the shifted Chebyshev polynomials of the third kind. Approximate analytical solution of FRDE is considered as Chebyshev series expansion. The fractional derivative is described in the Caputo sense. Using properties of Chebyshev polynomials FRDE with initial condition is reduced to a nonlinear system of algebraic equations which solved by the Newton iteration method. The accuracy and efficiency of the presented method is illustrated by numerical examples.
\end{abstract}

Keywords:Fractional Riccati differential equation, Shifted Chebyshev polynomials of the third kind, Chebyshev collocation method, Caputo fractional derivative

\section{Introduction}

Riccati differential equation has a great role in the mathematical modeling of several phenomena such as random processes, optimal control and diffusion problems [3, 4]. Recently, with the increasing importance of fractional derivative on interdisciplinary applications $[6,7$, $5,14,18]$ fractional Riccati differential equation has attracted much more attention of physicists and mathematicians.

Most fractional differential equations do not have exact solutions. Therefore, numerical methods have been proposed and investigated to get approximate solutions of Riccati differential equations of arbitrary order (FRDE). Jafari and Tajadodi [8] have presented He's variational iteration method to obtain the solution of FRDE. A fractional variational iteration method described in Riemann-Liouville derivative has been applied to the same problem in [16]. A numerical scheme which is combination of finite difference method and Pade'variational iteration has been studied by Sweliam et al. [23]. A stochastic technique has been developed for the solution of nonlinear Riccati differential equation of fractional order in [20]. Khader [9] has presented fractional Chebyshev finite difference method for FRDE. The 
spectral Laguerre collocation method has been presented by Khader et al. [10] for FRDE. A generalized Chebyshev wavelet operational matrix method has been proposed by Balaji [1]. New homotopy perturbation method has been presented by Khan et al. [12]. Chebyshev wavelet operational matrix of the fractional integration has been used to solve a nonlinear fractional differential equations[13].

In recent decades, the class of Chebyshev polynomials is one of the most useful polynomials in numerical analysis. These polynomials are used to solve integral equations and fractional order differential equations $[23,9,1,13,24,11,21,2]$. Let us consider the FRDE of the form:

$$
D^{\alpha} y(t)=a(t)+b(t) y(t)+c(t) y^{2}(t), t>0, \quad 0<\alpha \leq 1
$$

subject to the initial condition

$$
y(0)=c,
$$

where $a(t), b(t)$ and $c(t)$ are given functions, $c$ is an arbitrary constant, $D^{\alpha}$ is the Caputo fractional derivative operator of order $\alpha$. Our aim is to obtain an analytical approximate solution of Eq.(1) with initial condition (2) in terms of the shifted Chebyshev polynomials of the third kind.

Definition 1.1 : For $\mathrm{m}$ to be the smallest integer that exceeds $\alpha$ the Caputo time-fractional derivative operator of order $\alpha>0$ is defined as [19]

$$
D^{\alpha} f(x)=\frac{1}{\Gamma(m-\alpha)} \int_{0}^{x}(x-\tau)^{m-\alpha-1} \frac{d^{m} f(\tau)}{d \tau^{m}} d \tau,
$$

where $m-1<\alpha \leq m, m \in N, x>0$.

The linearity property of the Caputo fractional derivative is gives as

$$
D^{\alpha}(\lambda f(x)+\gamma g(x))=\lambda D^{\alpha} f(x)+\gamma D^{\alpha} g(x),
$$

where $\lambda$ and $\gamma$ are constants.

The organization of this paper is as follows: In Section 1, properties of Chebyshev polynomials of the third kind are given. In Section 3, numerical scheme is given to obtain an analytical approximate solution in terms of the shifted Chebyshev polynomials of the third kind of Eq. (1). In Section 4, numerical examples are pesented. Also a conclusion is given in Section 5. Note that numerical results have been computed by using Matlab programming.

\section{Some properties of Chebyshev polynomials of the third kind}

The Chebyshev polynomials $V_{n}(x)$ of the third kind are orthogonal polynomials of degree $n$ in $x$ defined on the $[-1,1][15]$ 


$$
V_{n}(x)=\frac{\cos \left(n+\frac{1}{2}\right) \theta}{\cos \frac{\theta}{2}},
$$

where $x=\cos \theta$ and $\theta \in[0, \pi]$. Also, these polynomials $V_{n}(x)$ are orthogonal on $[-1,1]$ with respect to the inner product

$$
<V_{n}(x), V_{m}(x)>=\int_{-1}^{1} \sqrt{\frac{x+1}{1-x}} V_{n}(x) V_{m}(x) d x=\left\{\begin{array}{l}
0, n \neq m \\
\pi, n=m
\end{array},\right.
$$

where $\sqrt{\frac{x+1}{1-x}}$ is weight function corresponding to $V_{n}(x)$ The polynomials $V_{n}(x)$ may be generated by using the recurrence

$$
V_{n+1}(x)=2 x V_{n}(x)-V_{n-1}(x), n=1,2, \ldots,
$$

with $V_{0}(x)=1, V_{1}(x)=2 x-1$.

Shifted Chebyshev polynomials of the third kind $V_{n}^{*}(x)$ of degree $n$ in $x$ on $[0,1]$ is given by $V_{n}^{*}(x)=V_{n}(2 x-1)$. These polynomials are orthogonal on the support interval $[0,1]$ as the following inner product:

$$
<V_{n}^{*}(x), V_{m}^{*}(x)>=\int_{0}^{1} \sqrt{\frac{x}{1-x}} V_{n}^{*}(x) V_{m}^{*}(x) d x=\left\{\begin{array}{l}
0, n \neq m \\
\frac{\pi}{2}, n=m
\end{array}\right.
$$

where $\sqrt{\frac{x}{1-x}}$ is weight function corresponding to $V_{n}^{*}(x)$ and normalized by the requirement that $V_{n}^{*}(1)=1$. The polynomials $V_{n}^{*}(x)$ may be generated by using the recurrence relations

$$
V_{n+1}^{*}(x)=2(2 x-1) V_{n}^{*}(x)-V_{n-1}^{*}(x), n=1,2, \ldots,
$$

with $V_{0}^{*}(x)=1, V_{1}^{*}(x)=4 x-3$.

The function $y(x)$ which belongs to the space of square integrable in $[0,1]$ may be expressed in terms of shifted Chebyshev polynomials of the third kind as

$$
y(x)=\sum_{i=0}^{\infty} b_{i} V_{i}^{*}(x)
$$

where the coeffcients $b_{i}$ are given by

$$
b_{i}=\frac{2}{\pi} \int_{0}^{1} \sqrt{\frac{x}{1-x}} V_{i}^{*}(x) d x
$$

Consider only the first $(N+1)$ terms of shifted Chebyshev polynomials of the third kind, so we can write 


$$
y_{N}(x)=\sum_{i=0}^{N} b_{i} V_{i}^{*}(x)
$$

Theorem 2.1. [22] Let $y_{N}(x)$ be approximated function in terms of shifted Chebyshev polynomials of the third kind as given in Eq.(3), suppose then, we obtain:

$$
D^{\alpha} y_{N}(x)=\sum_{i=\llbracket \alpha \rrbracket}^{N} \sum_{k=0}^{i-\llbracket \alpha \rrbracket} b_{i} M_{i, k}{ }^{(\alpha)} x^{i-k-\alpha},
$$

where $\llbracket \alpha \rrbracket$ is used to denote the smallest integer greater than or equal to $\alpha$ and

$$
M_{i, k}^{(\alpha)}=(-1)^{k} 2^{2 i-2 k} \frac{(2 i+1) \Gamma(2 i-k+1) \Gamma(i-k+1)}{\Gamma(k+1) \Gamma(2 i-2 k+2) \Gamma(i-k+1+\alpha)} .
$$

\section{Numerical scheme}

Assume that the solution of Eq.(1) with initial condition (2) can be written as

$$
y(t)=\sum_{i=0}^{N} b_{i} V_{i}^{*}(t)
$$

Substituting Eq.(5) into Eq.(1) and using Eq.(4) we have

$$
\sum_{i=\llbracket \alpha \rrbracket}^{N} \sum_{k=0}^{i-\llbracket \alpha \rrbracket} b_{i} M_{i, k}{ }^{(\alpha)} t^{i-k-\alpha}=a(t)+b(t) \sum_{i=0}^{N} b_{i} V_{i}^{*}(t)+c(t)\left(\sum_{i=0}^{N} b_{i} V_{i}^{*}(t)\right)^{2}
$$

Collocating Eq. (6) at $N$ points $t_{p}, p=1,2, \ldots, N$, we have:

$$
\sum_{i=\llbracket \alpha \rrbracket}^{N} \sum_{k=0}^{i-\llbracket \alpha \rrbracket} b_{i} M_{i, k}{ }^{(\alpha)} t_{p}^{i-k-\alpha}=a\left(t_{p}\right)+b\left(t_{p}\right) \sum_{i=0}^{N} b_{i} V_{i}^{*}\left(t_{p}\right)+c\left(t_{p}\right)\left(\sum_{i=0}^{N} b_{i} V_{i}^{*}\left(t_{p}\right)\right)^{2}
$$

For suitable collocation points we use roots of shifted Chebyshev polynomial of the third kinds $V_{N}^{*}(t)$. Also, substituting Eq.(5) into the initial condition (2) we can find

$$
\sum_{i=0}^{N} b_{i}(-1)^{i}(2 i+1)=c
$$

Eq.(7) and Eq.(8) give $N+1$ nonlinear algebraic equations. These nonlinear system can be solved by using Newton method in the unknown $b_{i}, i=0, \ldots, N$.

\section{Numerical experiments and comparison}

Example 4.1. Consider the following fractional Riccati differential equation:

$$
D^{\alpha} y(t)+y^{2}(t)=1, t>0, \quad 0<\alpha \leq 1
$$


subject to the initial condition $y(0)=0$. The exact solution of Eq.(9) is $y_{e}(t)=\frac{e^{2 t}-1}{e^{2 t}+1}$ when $\alpha=1$.

Let us consider $N=7$ for $\alpha=1$, and we have approximate analytical solution of Eq.(9) as follows:

$$
y(t)=\sum_{i=0}^{7} b_{i} V_{i}^{*}(t)
$$

From Eq.(7) we obtain

$$
\sum_{i=1}^{7} \sum_{k=0}^{i-1} b_{i} M_{i, k}{ }^{(1)} t_{p}{ }^{i-k-\alpha}=1-\left(\sum_{i=0}^{7} b_{i} V_{i}^{*}\left(t_{p}\right)\right)^{2},
$$

where $x_{p}$ are roots of the shifted Chebyshev polynomial $V_{7}^{*}(t)$, i.e.,

$x_{1}=0.98907380, \mathrm{x}_{2}=0.90450849, \mathrm{x}_{3}=0.75000000, \mathrm{x}_{4}=0.55226423$,

$\mathrm{x}_{5}=0.34549150, \mathrm{x}_{6}=0.16543469, \mathrm{x}_{7}=0.04322727$.

From Eq.(8) we have

$$
\sum_{i=0}^{N} b_{i}(-1)^{i}(2 i+1)=0
$$

Solving obtained nonlinear system (10)-(11) by Newton method at iteration 4, the unknown coeffcients are obtained as follows:

$b_{0}=0.61229667, b_{1}=0.17166657, \mathrm{~b}_{2}=-0.02189092, \mathrm{~b}_{3}=-0.00099910, \mathrm{~b}_{4}=0.000556$,

$b_{5}=-0.00002737, b_{6}=-0.00000983, b_{7}=0.00000148$.

The obtained numerical results by means of the proposed method are shown in Table 1, Table 2 and Fig. 1. Table 1 shows the approximate solutions of Eq.(9) obtained for $\alpha=1$. It contains the solution $y_{o}(t)$ obtained by our method for $N=7$; 10 , the solution $y_{v}(t)$ which is a polynomial with degree 7 obtained by variational iteration method (VIM) [16], the fourth component approximate solution $y_{h}(t)$ which is a polynomial with degree 7obtained by Modiffed Homotopy perturbation method [17], the solution $y_{s}(t)$ obtained by stochastic technique [20]. From the numerical results in Table1, it is clear that the obtained solution by our method for $N=7$ which is a polynomial with degree 7 is more close to the exact solution rather than other methods. For $N=10$ the solution $y_{o}(t)$ is exactly the same with the exact solution.

The exact solution of (9) for fractional order case is not available, however, approximate numerical solutions are available. For comparison the solution $y_{o}(t)$ given by the our method for $N=10$, reported approximate results of [16], [20] are given in Table 2 for $\alpha=0.5 ; 0.75$.

Figure 1 indicates the solution obtained by our method for $N=5$ which is a polynomial with degree 5, the solution obtained by variational iteration method (VIM) which is a polynomial 
with degree 7 [16] and the exact solution when $\alpha=1$. The results obtained by our method are more close to the exact solution.

Table 1. Comparison of results for the solution of the equation in Example 1 for $\alpha=1$.

\begin{tabular}{|c|c|c|c|c|c|c|}
\hline $\mathrm{t}$ & $y_{e}(t)$ & $y_{o}(t), N=7$ & $y_{o}(t), N=10$ & $y_{s}(t)$ & $y_{h}(t)$ & $y_{v}(t)$ \\
\hline 0 & 0 & 0 & 0 & $4.04 \times 10^{-9}$ & 0 & 0 \\
\hline 0.1 & 0.099667 & 0.099668 & 0.099667 & 0.099665 & 0.099668 & 0.099667 \\
\hline 0.2 & 0.197375 & 0.197375 & 0.197375 & 0.197400 & 0.197375 & 0.197375 \\
\hline 0.3 & 0.291312 & 0.291313 & 0.291312 & 0.291351 & 0.291312 & 0.291320 \\
\hline 0.4 & 0.379948 & 0.379949 & 0.379948 & 0.379954 & 0.379944 & 0.380005 \\
\hline 0.5 & 0.462117 & 0.462117 & 0.462117 & 0.462081 & 0.462078 & 0.462375 \\
\hline 0.6 & 0.537049 & 0.537049 & 0.537049 & 0.537021 & 0.536857 & 0.537923 \\
\hline 0.7 & 0.604367 & 0.604368 & 0.604367 & 0.604390 & 0.603631 & 0.606768 \\
\hline 0.8 & 0.664036 & 0.664037 & 0.664036 & 0.664095 & 0.661706 & 0.669695 \\
\hline 0.9 & 0.716297 & 0.716298 & 0.716297 & & 0.709919 & 0.728139 \\
\hline 1 & 0.761594 & 0.761594 & 0.761594 & 0.76159 & 0.746032 & 0.784126 \\
\hline
\end{tabular}

Table 2. Comparison of results for the solution Example 1 for fractional order cases

\begin{tabular}{|c|c|c|c|c|c|c|}
\hline $\mathrm{t}$ & $y_{v}(t), \alpha=0.5$ & $y_{v}(t), \alpha=0.75$ & $y_{v}(t), \alpha=0.5$ & $y_{v}(t), \alpha=0.75$ & $y_{o}(t), \alpha=0.5$ & $y_{o}(t), \alpha=0.75$ \\
\hline 0 & 0.009493 & 0.000798 & 0 & 0 & 0 & 0 \\
\hline 0.1 & 0.289667 & 0.165087 & 0.086513 & 0.190102 & 0.326538 & 0.187449 \\
\hline 0.2 & 0.386489 & 0.276350 & 0.427831 & 0.161584 & 0.432390 & 0.307255 \\
\hline 0.3 & 0.441120 & 0.356196 & 0.405062 & 0.500536 & 0.505013 & 0.402926 \\
\hline 0.4 & 0.482348 & 0.416916 & 0.321523 & 0.483479 & 0.550349 & 0.479914 \\
\hline 0.5 & 0.516379 & 0.465520 & 0.413682 & 0.550470 & 0.590155 & 0.543708 \\
\hline 0.6 & 0.544872 & 0.506004 & 0.515445 & 0.610344 & 0.621147 & 0.596804 \\
\hline 0.7 & 0.568545 & 0.540629 & 0.626403 & 0.666961 & 0.643440 & 0.640819 \\
\hline 0.8 & 0.587895 & 0.570632 & 0.745278 & 0.723760 & 0.665904 & 0.678077 \\
\hline 0.9 & 0.603344 & 0.596636 & 0.870074 & 0.783638 & 0.682599 & 0.709518 \\
\hline 1 & 0.615268 & 0.618873 & 0.998176 & 0.848783 & 0.698172 & 0.736279 \\
\hline
\end{tabular}




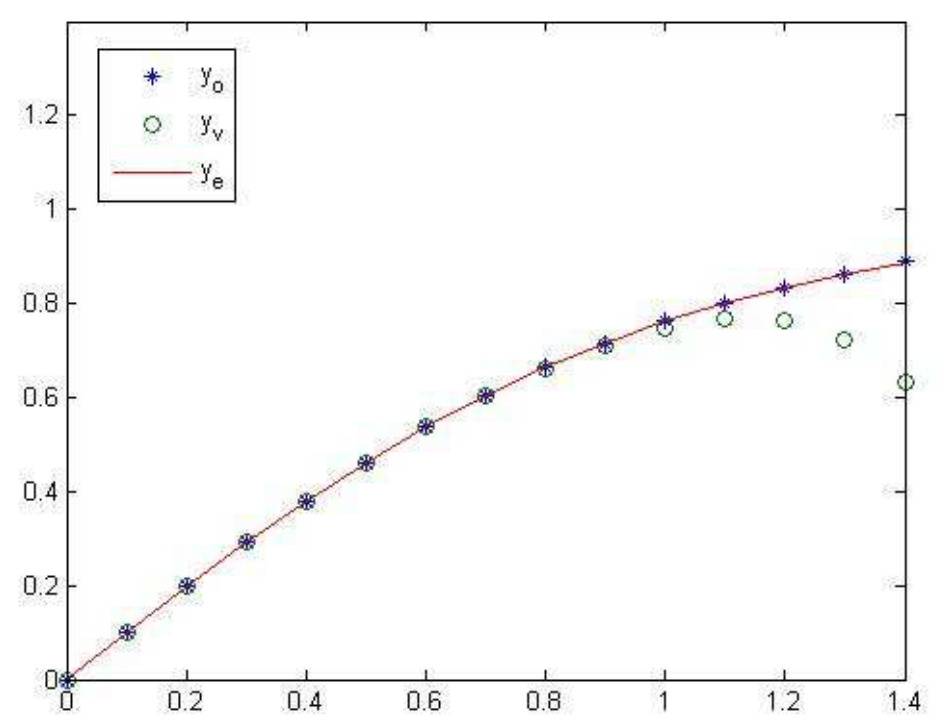

Figure 1: The behavior of the approximate solutions and the exact solution in Example 1 for $\alpha=1$.

Example 4.2. Consider the following fractional Riccati differential equation:

$$
D^{\alpha} y(t)+-2 y(t)+y^{2}(t)=1, t>0, \quad 0<\alpha \leq 1
$$

subject to the initial condition $y(0)=0$. The exact solution of Eq.(9) is $y_{e}(t)=1+\sqrt{2} \tanh (t \sqrt{2})+\frac{1}{2} \log \left(\frac{\sqrt{2}-1}{\sqrt{2}+1}\right)$ when $\alpha=1$.

Using the suggested method, the obtained numerical results are given in Fig. 2, Fig. 3, Table 3 and Table 4. Figure 2 indicates the solution $y_{o}(t)$ of Eq.(12) obtained by our method for $N=7$, the solution $y_{n h}(t)$ which is a polynomial with degree 21 obtained by new homotopy perturbation method [12] and the exact solution $y_{e}(t)$ for $\alpha=1$. As shown in Figure 2 the approximate solutions $y_{o}(t)$ and $y_{n h}(t)$ are in high agreement with the exact solutions in the interval $[0,1]$. After $t=1$ while the approximate solution $y_{n h}(t)$ moves away from the exact solution, the approximate solution $y_{o}(t)$ shows the same behavior with the exact solution.

Figure 3 indicates the solution $y_{o}(t)$ of Eq.(12) obtained by our method for $\alpha=0.5 ; 0.75 ; 1$ for $N=7$ and the exact solution for $\alpha=1$.

Table 3 indicates the approximate solutions for Eq.(12) obtained for $\alpha=1$ on the [0,1]. It presents the solution $y_{o}(t)$ obtained by our method for $N=10$; the solution $y_{c}(t)$ which contains 192 term obtained by Chebyshev wavelets method [13], the solution $y_{v}(t)$ which is a polynomial with degree 7 obtained by fractional variational iteration method [16], the solution $y_{s}(t)$ obtained by stochastic technique [20], the solution $y_{n h}(t)$ which is a polynomial with degree 21 obtained by new homotopy perturbation method [12]. From the numerical results in 
Table 3, it is clear that the obtained numerical solution by using our method is in very good agreement with the exact solution when $\alpha=1$.

The exact solution of (12) for fractional order case is not available. However, approximate numerical solutions are available. Table 4 indicates the approximate solutions for Eq.(12) obtained for $\alpha=0.5$ on the [0,1]. It presents the solution $y_{o}(t)$ obtained by our method for $N=$ 10; the solution $y_{c}(t)$ which contains 192 term obtained by Chebyshev wavelets method [13], the solution $y_{v}(t)$ which is a polynomial with degree 7 obtained by fractional variational iteration method [16], the solution $y_{s}(t)$ obtained by stochastic technique [20], the solution $y_{n h}(t)$ which is a polynomial with degree 21 obtained by new homotopy perturbation method [12].

Table 3. Comparison of results for the solution of the equation in Example 2 for $\alpha=1$.

\begin{tabular}{|c|l|c|c|c|c|c|}
\hline $\mathrm{t}$ & $y_{e}(t)$ & $y_{o}(t)$ & $y_{c}(t)$ & $y_{n h}(t)$ & $y_{s}(t)$ & $y_{v}(t)$ \\
\hline 0 & 0 & 0 & 0 & 0 & $6.58 \times 10^{-9}$ & 0 \\
\hline 0.1 & 0.110295 & 0.110295 & 0.110311 & 0.110295 & 0.110328 & 0.110266 \\
\hline 0.2 & 0.241977 & 0.241977 & 0.241995 & 0.241977 & 0.241997 & 0.241585 \\
\hline 0.3 & 0.395105 & 0.395105 & 0.395123 & 0.395105 & 0.395101 & 0.393515 \\
\hline 0.4 & 0.567812 & 0.567812 & 0.567829 & 0.567812 & 0.567797 & 0.564013 \\
\hline 0.5 & 0.756014 & 0.756015 & 0.756029 & 0.756014 & 0.756008 & 0.749528 \\
\hline 0.6 & 0.953566 & 0.953566 & 0.953576 & 0.953566 & 0.953580 & 0.945155 \\
\hline 0.7 & 1.152949 & 1.152949 & 1.152955 & 1.152952 & 1.152973 & 1.144826 \\
\hline 0.8 & 1.346364 & 1.346364 & 1.346365 & 1.346427 & 1.346374 & 1.341552 \\
\hline 0.9 & 1.526911 & 1.526911 & 1.526909 & 1.527752 & 1.526890 & 1.527690 \\
\hline 1 & 1.689498 & 1.689498 & 1.689494 & 1.697811 & 1.689459 & 1.695238 \\
\hline
\end{tabular}

Table 4. Comparison of results for the solution of the equation in Example 2 for $\alpha=0.5$.

\begin{tabular}{|c|l|c|c|c|c|}
\hline $\mathrm{t}$ & $y_{o}(t)$ & $y_{c}(t)$ & $y_{s}(t)$ & $y_{n h}(t)$ & $y_{v}(t)$ \\
\hline 0 & 0 & 0 & 0.009420 & 0 & 0 \\
\hline 0.1 & 0.580257 & 0.592756 & 0.574648 & 0.406052 & 0.577431 \\
\hline 0.2 & 0.921636 & 0.9331796 & 0.890890 & 0.646694 & 0.912654 \\
\hline 0.3 & 1.168259 & 1.1739836 & 1.090716 & 0.879747 & 1.166253 \\
\hline 0.4 & 1.340087 & 1.3466546 & 1.230069 & 1.109343 & 1.353549 \\
\hline 0.5 & 1.470003 & 1.4738876 & 1.334181 & 1.328067 & 1.482633 \\
\hline 0.6 & 1.568290 & 1.5705716 & 1.415512 & 1.524645 & 1.559656 \\
\hline 0.7 & 1.642899 & 1.646199 & 1.480918 & 1.687863 & 1.589984 \\
\hline 0.8 & 1.705323 & 1.706880 & 1.534604 & 1.809799 & 1.578559 \\
\hline 0.9 & 1.754742 & 1.756644 & 1.579396 & 1.890285 & 1.530028 \\
\hline 1 & 1.796803 & 1.798220 & 1.617332 & 1.958464 & 1.448805 \\
\hline
\end{tabular}




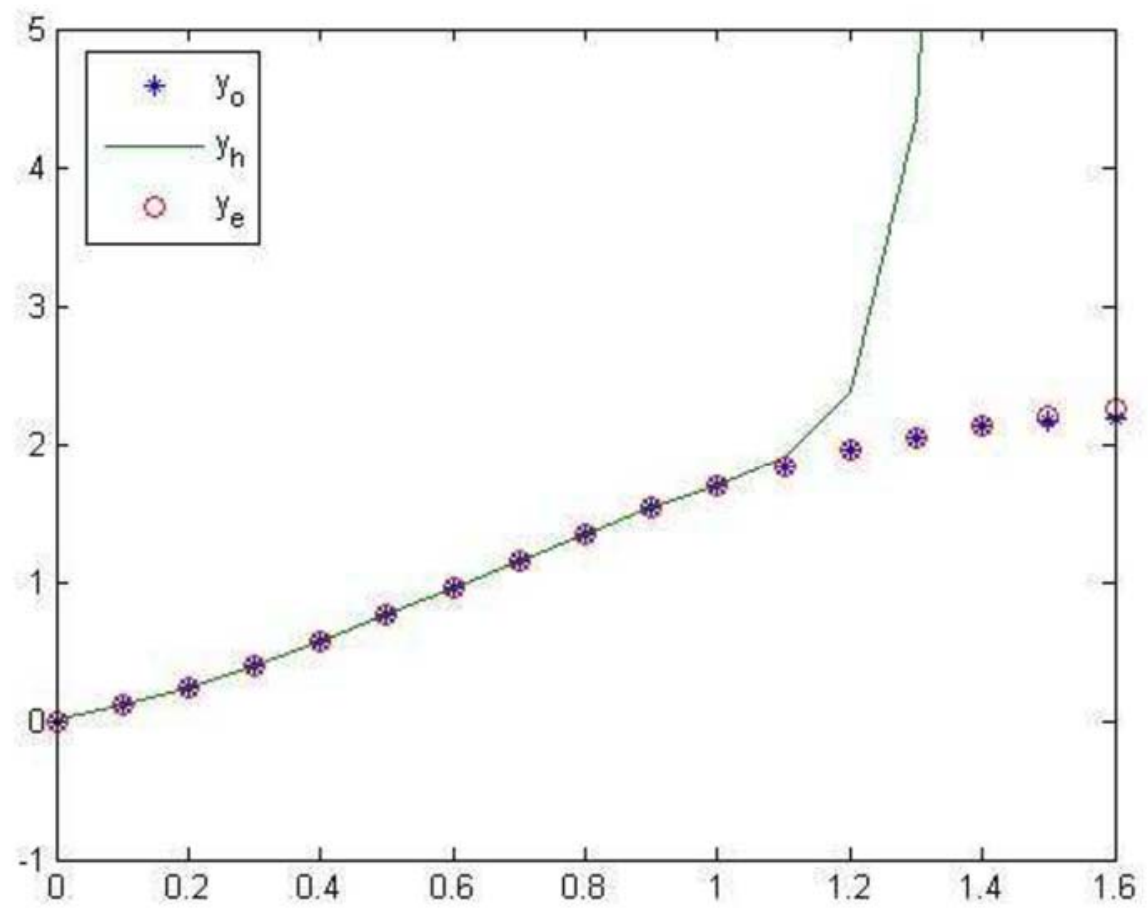

Figure 2. The behavior of the approximate solutions and the exact solution in Example 2 for $\alpha=1$.

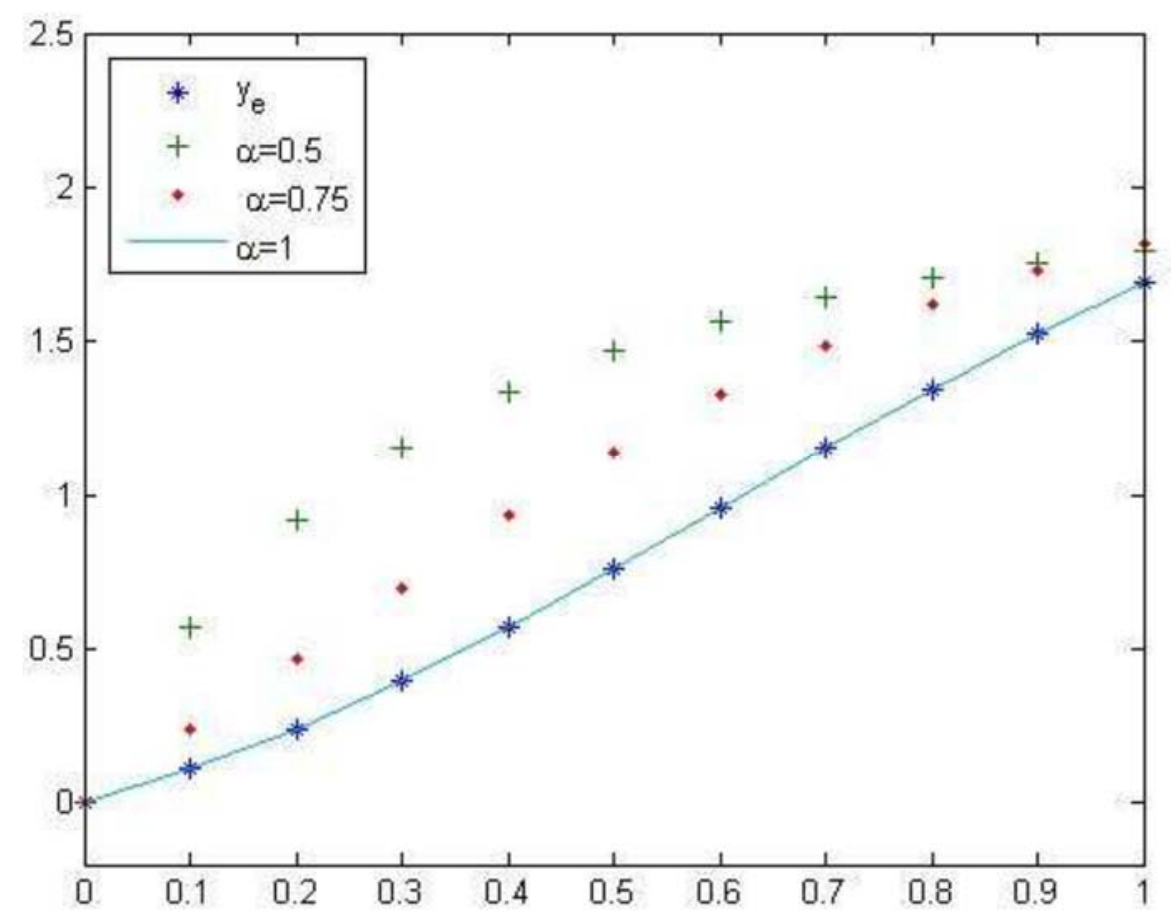

Figure 3. The behavior of the approximate solution with different values of $\alpha$ and the exact solution in Example 2. 


\section{Conclusion}

In this paper, FRDE is reduced to a nonlinear system of algebraic equations by using the shifted Chebyshev polynomials of the third. The obtained algebraic equations are solved by using Newton method. The solutionis expressed as a truncated Chebyshev series and so it can be easily computed for arbitrary values of $t$ using any computer programming in a short time without any computational effort. The numerical results show that the algorithm converges as the number of $N$ terms is increased.

The accuracy of the method are shown by some numerical examples. The obtained results are compared with the results from the other methods. From the numerical results, it is obvious that the proposed method provides better accuracy and efficiency than other methods.

\section{References}

[1] Balaji S. "Solution of nonlinear Riccati differential equation using Chebyshev wavelets.”WSEAS Trans. Math. 13 (2014): 441 - 451.

[2] Dalir M. and Bashour M. “Applications of fractional calculus.” Appl. Math.Sci. 4 (2010): 1021 - 1032.

[3] Einicke G.A., White L.B. and Bitmead R.R. "The use of fake algebraic Riccati equations for co-channel demodulation.” IEEE Trans. Signal Process 51 (2003): 2288 - 2293.

[4] Gerber M., Hasselblatt N. and Keesing D. "The Riccati equation: pinching of forcing and solutions.”Experiment Math. 12 (2003): 129 - 134.

[5] Grigorenko I. and Grigorenko I.E. "Chaotic dynamics of the fractional Lorenz system." Phys. Rev. Lett. 91 (2003): 034101 - 034104.

[6] He J.H. "Some applications of nonlinear fractional di_erential equationsand their approximations." Bull. Sc. Technol. 15 (1999): 86 - 90.

[7] He J. H. "Approximate analytical solution for seepage flow with fractional derivatives in porous media." Comput. Methods. Appl. Mech Eng. 167 (1998) : 57 - 68.

[8] Jafari H. and Tajadodi H. "He's Variational Iteration Method for Solving Fractional Riccati Differential Equation." J. Differ. Equations 2010 (2010): 1 - 8.

[9] Khader M.M. " Numerical treatment for solving fractional Riccati differential equation,." J. Egyptian Math. Soc. 21 (2013): 32 - 37.

[10] Khader M.M., Mahdy A.M.S. and Mohamed E.S. " On Approximate Solutions For Fractional Riccati Differential Equation." J. Eng. Appl. Sci. (2014) : 2683 - 2689.

[11] Khader M.M. "On the numerical solutions for the fractional diffusion equation." Commun. Nonlinear Sci. Nume. Simulat. 16 (2011): 2535 -2542.

[12] Khan N.A., Ara A. and Muhammad J. "An effcient approach for solving the Riccati equation with fractional orders." Comput. Math. Appl. 61 (2011): 2683 - 2689.

[13] Li Y. "Solving a nonlinear fractional differential equation using Chebyshev wavelets." Commun. Nonlinear Sci. Numer. Simulat. 15 (2010): 2284- 2292.

[14] Mainardi F. Fractional calculus: Some basic problems in continuum and statistical mechanics, In: A. Carpinteri, F. Mainardi, Editors, Fractals and Fractional Calculus in Continuum Mechanics, Springer-Verlag, NewYork, 1997, 291 - 348. 
[15] Mason J.C. and Handscomb D.C. Chebyshev Polynomials, Chapman and Hall, CRC, New York, NY, Boca Raton, 2003.

[16] Merdan M. "On the Solutions Fractional Riccati Differential Equation with Modified Riemann-Liouville Derivative." J. Differ. Equations 2012 (2012): 1 - 17.

[17] Odibat Z. and Momani S. "Modifed homotopy perturbation method: application to quadratic Riccati differential equation of fractional order." Chaos Soliton. Fract. 36 (2008): 167 - 174.

[18] Podlubny I. "Geometric and physical interpretation of fractional integration and fractional differentiation." Fract Calculus App. Anal. 5 (2002): 367 - 386.

[19] Podlubny I., Fractional differential equations, Academic press, NewYork, 1999.

[20] Raja M.A.Z., J. A. Khan J.A. and Qureshi I.M. "A new stochastic approach for solution of Riccati differential equation of fractional order." Ann. Math.Artif. Intell. 60 (2010): $229-250$.

[21] Sousa E. "Numerical approximations for fractional diffusion equation via splines. " Compu.t Math. App. 62 (2011): 983 - 944.

[22] Sweilam N.H., Nagy A.M. and El-Sayed A. "On the numerical solution of space fractional order diffusion equation via shifted Chebyshev polynomials of the third kind,." J. King Saud Univ. Sci. 28 (2016): 41-47.

[23] Sweilam N.H., Khader M.M. and Mahdy A.M.S. "Numerical Studies for Solving Fractional Riccati Differential Equation." Appl. Appl. Math. 7 (2012): 595 - 608.

[24] Tadjeran C., Meerschaert M. and H.P. Scheffler. "A second-order accurate numerical approximation for the fractional diffusion equation." J. Comput. Phys. 21 (2006): 205 213. 